\title{
TWO OBJECTIVE PUBLIC SERVICE SYSTEM DESIGN PROBLEM
}

\author{
Jaroslav Janáček, Michal Koháni, Dobroslav Grygar*, René Fabricius \\ Faculty of Management Science and Informatics, University of Zilina, Zilina, Slovakia \\ *E-mail of corresponding author: dobroslav.grygar@fri.uniza.sk
}

\section{Resume}

The public service system serves population spread over a geographical area from a given number of service centers. One of the possible approaches to the problem with two or more simultaneously applied contradicting objectives is determination of the so-called Pareto front, i.e. set of all the feasible non-dominated solutions. The Pareto front determination represents a crucial computational deal, when a large public service system is designed using an exact method. This process complexity evoked an idea to use an evolutionary metaheuristic, which can build up a set of non-dominated solution continuously in the form of an elite set. Nevertheless, the latter approach does not assure that the resulting set of solutions represents the true Pareto front of the multi-objective problem solutions. Within this paper, authors deal with both approaches to evaluate the difference between the exact and heuristic approaches.

Available online: https://doi.org/10.26552/com.C.2021.4.E68-E75
Article info

Received 17 December 2020

Accepted 15 February 2021

Online 21 September 2021

\section{Keywords:}

service system, multi-objective, Pareto front, exact approach, genetic algorithm

ISSN 1335-4205 (print version) ISSN 2585-7878 (online version)

\section{Introduction}

The public service system design problem is usually formulated as a task of selection of $p$-center locations from a finite set of possible center location so that a given objective, based on user's distances to the nearest service center, is minimal. According to applied objective, the design problem can be formulated in several ways. The most usual one is the way that prefers the solution with minimal average user's distances to the service center. This objective is called the min-sum or system objective and it has been used by many authors in emergency system designing. In this case, the problem is often presented as the weighted p-median problem [1-4]. This min-sum problem consists of determination of the p-center locations so that the sum of user's distances to the nearest service center, weighted by an expected number of the user demands, is minimal [5-6].

In contrast to the min-sum formulations, users of a public service system share cost of the system by paying tax, which approves them to claim the equal or fair access to the provided service. A plethora of fairness schemes was studied in connection with public service system design. The most known one is the min-max objective, where the maximal distance from a user to the nearest service center should be minimal. This formulation of the public service design is called the $\mathrm{p}$ - center problem. The strongest fairness scheme, applicable in the public service system design, is the so-called lexicographic min-max criterion [7]. When solving a fair public system design problem as the lexicographical min-max fair design, a sequential process of optimization is used. The nearest distance to the service center of the worst situated user is minimized first, followed by the minimizing of the distance of the second-worst situated users and so on, unless the minimal reached distance of the previously processed users is worsened.

All the above-mentioned formulations of public service system design have only one objective function and can be efficiently solved by the so-called radial approach in the case of the min-sum formulation [4, 8-10]. The min-max fair public service system design problem proved to be easily solvable using the radial formulation [11]. The radial formulation has been also successfully applied to the lexicographical min-max fair design [12].

When consequences of the fairness schemes were studied, it was found that the fair and system objectives contradict. It means that if a fair objective is minimized, then the system objective takes bigger, i.e. worse, value, what usually means that the fairer solution is paid by worsening of the average distance between a user and the service center [13-14]. The similar relationship can be observed between the sum of fixed charges and the cost of users' demand satisfaction [15]. The next case of the objective contradiction can be found in the field of fuzzy set theory applied to the public service system design in the situations, when traversing times in a transportation network are uncertain [16]. In such a case, the level of satisfaction contradicts to the min-sum objective function value [17]. 
All the mentioned cases represent a mathematically unsolvable situation because there exists a set of feasible solutions with different values of the objective functions and it is impossible to decide on the best one without an additional comparative rule. A possible approach to the public service system design with two or more contradicting objectives consists in producing Pareto front, or a set of non-dominated solutions. This output of the designing process enables to put the final decision on a supervising manager's board.

The real Pareto front is a set of all the nondominated solutions, what means that each other solution, is outperformed by a solution of the front in each of the applied objectives. As the most of the public service system design problems have finite domains of objective functions, the real Pareto front can be obtained by a finite sequence of runs of an exact mathematical programming method. This approach represents a crucial computational deal, especially when a large public service system is designed using an exact mathematical programming method. It is necessary to consider that each run of the sequence represents solving of a hard and large combinatorial problem, which solution is obtained by time demanding inspection of a vast searching tree. To avoid this computational burden, professionals prefer a heuristic approach based on imitating the biological processes [18-19]. To obtain a good set of the non-dominated feasible solutions, an evolutionary metaheuristic seems to be a convenient tool, because an evolutionary metaheuristic is able to build up a set of the non-dominated solutions continuously in the form of an elite set [20]. Nevertheless, it must be taken into account that the latter approach does not assure that the resulting set of solutions represents the true Pareto front of the multi-objective problem solutions.

Within this paper, authors studied both approaches and compared them from the points of computational time and quality of the resulting set of non-dominated solutions. The solved cases of the public service system design will dispose of with min-sum and min-max objectives.

The remainder of the paper is organized as follows: Section 2 is devoted to the radial formulation of the min-sum problem with limited min-max criterion. The exact approach to Pareto front determination is described in Section 3 and metaheuristic approach is explained in Section 4. The associated numerical experiments are reported in Section 5. The results and findings are summarized in Section 6.

\section{Radial formulation of the min-sum problem with the limited distance}

The studied public service system is designed to satisfy demands for service of the system users, which are in the serviced area at finite set $J$ of user locations. The demand of a user $j \in J$ is expressed by an expected frequency $b_{j}$ of visits performed by a servicing vehicle. It is assumed that the vehicles start their servicing route from a service center and they return back to their center after a user's demand has been satisfied. The public service system design consists of locating of the $p$ service centers at the possible service center locations, which form a finite set $I$. The further defined objectives are based on integer network distances, where symbol $d_{i j}$ denotes the network distance from location $i \in I$ to the location $j \in J$ for any pair of locations from $I$ and $J$. The min-sum objective function value $f^{m S}\left(I_{1}\right)$ for a given set $I_{1}$ of located service centers can be defined by:

$f^{m S}\left(I_{1}\right)=\sum_{j \in J} b_{j} \min \left\{d_{i j}: i \in I_{1}\right\}$.

The min-max objective function value $f^{n M}\left(I_{p}\right)$ of the same design $I_{1}$ of the public service system is given by:

$f^{m M}\left(I_{1}\right)=\max \left\{\min \left\{d_{i j}: i \in I_{1}\right\}: j \in J\right\}$.

The public service system design problem, with the min-sum objective and with limit $h$ of any distance between a user and the associated service center, can be modelled by the combinatorial model in Equation (3).

$\min \left\{f^{m S}\left(I_{1}\right): I_{1} \subset I,\left|I_{1}\right|=p, f^{m M}\left(I_{1}\right) \leq h\right\}$.

The problem in Equation (3) can be rewritten into a linear programming form in several ways and then the problem can be solved by any integer programing solver. Based on our previous experiences, we describe the problem by a radial model, which proved to be more easily solvable than the other models.

Let $m$ denote the maximal relevant distance between a possible service center location and a user location. Then the following three-dimensional matrix $\left\{a_{i j s}\right\}$ can be defined by Equation (4) for each $i \in I, j \in J$ and $s=0$, ..., $m-1$.

if $d_{i j} \leq s$ then $a_{i j s}=1$, else $a_{i j s}=0$.

To complete the model, the decision variables $y_{i} \in\{0,1\}$ for $i \in I$ are introduced. The variable $y_{i}$ models the decision on service center location at place $i \in I$. The variable takes the value of 1 if a service center is located at $i$ and it takes the value of 0 otherwise. Further, the auxiliary zero-one variables $x_{j s}$ for $j \in J$ and $s=0, \ldots, h-1$ are introduced. The variable $x_{j s}$ takes the value of 1 , if the distance from a user located at $j \in J$ to the nearest located center is greater than $s$ and it takes the value of 0 otherwise. Then, the problem in Equation (3) can be formulated as follows.

Minimize $\sum_{j \in J} b_{j} \sum_{s=0}^{h-1} x_{j s}$.

Subject to: $x_{j s}+\sum_{i \in I} a_{i j s} y_{i} \geq 1$

for $j \in J, s=0, \ldots, h-1$ 


$$
\begin{aligned}
& \sum_{i \in I} y_{i}=p \\
& \sum_{i \in I} a_{i j h} y_{i} \geq 1 \text { for } j \in J \\
& y_{i} \in\{0,1\} \text { for } i \in I \\
& x_{j s} \geq 0 \text { for } j \in J, s=0,1, \ldots, h-1 .
\end{aligned}
$$

In this model, the objective function in Equation (5) represents the total travel distance necessary for satisfaction of all the users' demands, as the expression $x_{j 0}+x_{j 1}+x_{j 2}+\ldots+x_{j h-1}$ constitutes the distance to user location $j$ from the nearest located service.

The constraints in Equation (6) ensure that the variables $x_{j s}$ can take the value of 0 , if there is at least one center located in radius $s$ from the user location $j$ and constraint in Equation (7) limits the number of located centers by $p$. The constraints in Equation (8) ensure that each user has a service center in the radius $h$.

Let $I_{R}(h)$ denote the resulting set of service center locations $\left\{i \in I: y_{i}^{*}=1\right\}$ obtained from optimal solution $\left(\boldsymbol{y}^{*}, \boldsymbol{x}^{*}\right)$ of the problem in Equations (5)-(10) for a given integer value $h$.

\section{The Pareto front determination using the exact approach}

Quality of any feasible solution $I_{1}$, of the abovementioned public service design problem, can be evaluated according to each of the two contradicting objectives, $f^{n S}\left(I_{1}\right)$ and $f^{n M}\left(I_{1}\right)$, described by (1) and (2), respectively. A solution $I_{1}^{*}$ is called the non-dominated if there exists no feasible solution $I_{1}$ such that $f^{n S}\left(I_{1}\right) \leq$ $f^{n S}\left(I_{1}{ }^{*}\right)$ and $f^{n M}\left(I_{1}\right) \leq f^{n M}\left(I_{1}^{*}\right)$ and, in addition, if it holds either $f^{m S}\left(I_{1}\right)<f^{m S}\left(I_{1}^{*}\right)$ or $f^{n M}\left(I_{1}\right)<f^{m M}\left(I_{1}^{*}\right)$. The real Pareto front is a set of all the non-dominated solutions. As the set of all the feasible solutions is finite, the Pareto front of the studied problem must be also finite and it can be obtained by the process described below.

Let the function $f^{n S}\left(I_{R}(h)\right)$ be generalized so that it gives the value bigger than a penalty $Q$, when the problem has no feasible solution for given $h$. It is asserted that the Pareto front solutions can be selected from the sequence of solutions $I_{R}(h)$ for $h=h_{0}, \ldots, m$, where $h_{0}$ is the lowest integer, for which $f^{m S}\left(I_{R}\left(h_{0}\right)\right)<Q$ holds, i.e. $I_{R}\left(h_{0}\right)$ is a feasible solution of the problem in Equation (3).

Obviously, the solution $I_{R}\left(h_{0}\right)$, evaluated by a pair of the two objective values $\left[f^{n S}\left(I_{R}\left(h_{0}\right)\right), f^{m M}\left(I_{R}\left(h_{0}\right)\right)\right]$, is a non-dominated solution of the public service system design problem from the following reasons. In this case, the equality $h_{0}=f^{m M}\left(I_{R}\left(h_{0}\right)\right)$ holds, since no feasible solution exists for $h<h_{0}$ and thus $f^{n M}\left(I_{R}\left(h_{0}\right)\right) \leq f^{n M}\left(I_{1}\right)$ holds for each feasible solution $I_{1}$. As $I_{R}\left(h_{0}\right)$ is optimal solution of Equation (3) for $h_{0}=f^{n M}\left(I_{R}\left(h_{0}\right)\right)$, then each feasible solution $I_{1}$ satisfying $h_{0}=f^{n M}\left(I_{1}\right)$ also satisfies the following inequality $f^{m S}\left(I_{1}\right) \geq f^{m S}\left(I_{R}\left(h_{0}\right)\right)$.
Further, the elements of the sequence $\left\{I_{R}(h): h=h_{0}\right.$, $\ldots, m\}$ must generally satisfy Equation:

$$
\begin{aligned}
& f^{m S}\left(I_{R}(h)\right) \geq f^{m S}\left(I_{R}(h+1)\right) \text { and } \\
& f^{m M}\left(I_{R}(h)\right) \leq f^{m M}\left(I_{R}(h+1)\right) \text { for } \\
& h=h_{0}, \ldots, m-1 .
\end{aligned}
$$

It is noted that if $f^{n S}\left(I_{R}(h)\right)=f^{n S}\left(I_{R}(h+1)\right)$, then $I_{R}(h+1)$ cannot be the new non-dominated solution, because it is either dominated by $I_{R}(h)$ or it has the same evaluation in both objective functions.

One can also prove that the implication in Equation (12) holds for the sequence $\left\{I_{R}(h): h=h_{0}, \ldots, m\right\}$.

$$
\begin{aligned}
& \text { if } f^{m S}\left(I_{R}(h)\right)>f^{m S}\left(I_{R}(h+1)\right), \text { then also } \\
& f^{m M}\left(I_{R}(h)\right)<f^{m M}\left(I_{R}(h+1)\right) \text { for } \\
& h=h_{0}, \ldots, m-1 .
\end{aligned}
$$

The implication can be proved by contradiction. Let it be supposed that the assumption holds and that $f^{n M}\left(I_{R}(h)\right)=f^{n M}\left(I_{R}(h+1)\right)$. It means that the increase of $h$ does not impact the optimal solution of Equation (3), i.e. no distance from a user to the nearest service center exceeds $f^{n M}\left(I_{R}(h)\right)$. It follows that $f^{n S}\left(I_{R}(h)\right)=f^{n S}\left(I_{R}(h+1)\right)$, what contradicts the assumption.

Now, one can claim that if $f^{n S}\left(I_{R}(h)\right)>f^{n S}\left(I_{R}(h+1)\right)$, then $I_{R}(h+1)$ represents a new element of the Pareto front. The claim follows from the facts that $I_{R}(k)$ for $k<h+1$ does not dominate $I_{R}(h+1)$ and no solution $I_{R}(k)$ for $k>h+1$ can dominate $I_{R}(h+1)$ due to Equation (12).

Based on the above reasons, one can apply the following algorithm to establish the Pareto front for the solved problem.

0 . Determine $h_{0}$ and insert $I_{R}\left(h_{0}\right)$ into the Pareto front. Set $h=h_{0}+1$.

1. While $h \leq m$ repeat step 2 .

2. Compute $I_{R}(h)$.

If $f^{m S}\left(I_{R}(h)\right)<f^{n S S}\left(I_{R}(h-1)\right)$, then insert $I_{R}(h)$ into the Pareto front.

Set $h=h+1$.

\section{The genetic algorithm for the set of non- dominated solutions}

The genetic algorithm (GA) became popular in solving large combinatorial optimization problems. The main idea comes from the theory of evolution. In nature, populations exchanges are performed by transition and exchange of genetic information between members of a current population. This process is modelled using algorithmic operations of crossovers and mutations. The GA is suitable for solving problems where a solution can be represented by vector $\mathbf{y}$ with $0-1$ components. A solution can be also called a member of a population or individual. This vector can be called chromosome and its components are called genes. Chromosomes modelled in this way are changed by implemented operations of 


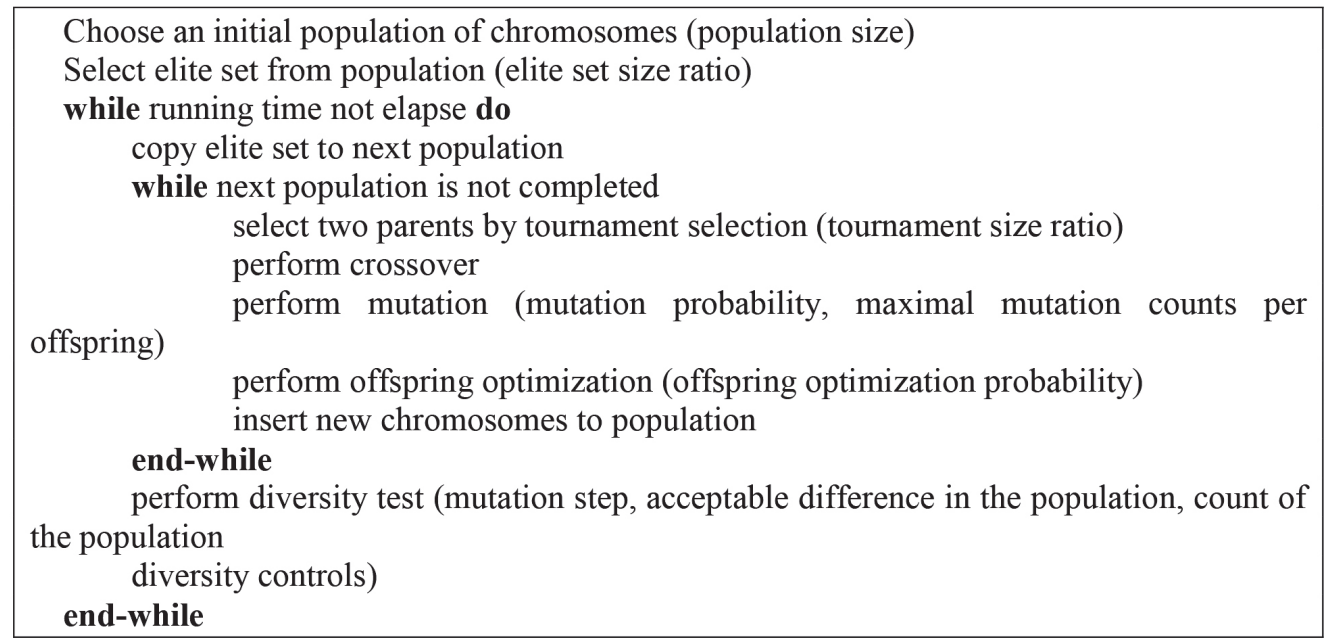

Figure 1 Used template of genetic algorithm with important parameters [21]

mutation and crossover, like the natural process. The mutation changes the value of one or more components in chromosome with some probability. Crossover is the process of generating two offspring from two-parent chromosomes [21]. The set of newly created individuals represents a pool of candidates for the next population. The new population is formed using the approach where members of the elite set from the old population are transited to the new population and new population is created by selection of individuals from the pool.

In authors' previous research, the genetic algorithm was implemented as metaheuristic for the given problem. As mentioned above, the set of good solutions is evaluated by the two partially conflicting criteria in the problem. As a result, a collection of good solutions is preferred rather than the single best solution. That implementation yields the semi-Pareto front as the output of an optimization run. The semi-Pareto front, obtained by the heuristic, approximates the exact Pareto front. The semi Pareto front is a part of the elite set. Adjustment of this algorithm to the goal of obtaining the good semi-Pareto front is based on testing if the obtained solution should be added to the current semi-Pareto front. here is briefly introduced The basic structure of the implemented genetic algorithm, is here briefly introduced, in the form of a template shown in Figure 1 [21].

For the better explanation of the implemented algorithm the specific modifications, performed in the introduced steps of the template, are described. It is decided to represent a solution using a list of the $p$-locations instead of $0-1$ vector. The $0-1$ vector can be obtained by a simple conversion if necessary. The initial population is generated randomly. For this implementation, the special crossover and mutation were developed $r$ to preserve the admissibility of the created solutions. That implementation of the crossover takes advantage of the fact that the number of differing genes in the two selected solutions is always even. Genes (possible located centres) that are the same in both parents are preserved in both offspring. For the set of differing genes, the associated portion of units is distributed randomly over the offspring so that each offspring gets the same number of units. This approach ensures that the offspring are also feasible solutions. Mutation process is also implemented with regarding the offspring feasibility. The mutation is a swap between one selected gene and the non-selected one.

One iteration of the population change works as follows. Individuals from the old elite set remain as individuals of the new population. To complete the new generation, the next individuals are obtained by crossover and selection from the previous population.

Pairs of individuals are selected from the previous generation by the random process called the tournament. It consists of random selection of individuals based on their fitness. This process is repeated until the population is complete. After that, the semi-Pareto front and elite set are updated in the following way. Each new member of the population is tested whether it belongs to the semi-Pareto front. If yes, it is inserted in it, dominated ones are removed. Then the process continues with the next iteration.

Tuning of the GA parameters is an important part of this study. Information on the GA parameters are given in the next Section.

\section{Computational study}

The multiple tests were performed to compare the metaheuristic approach with the exact results. All the heuristic tests were done on the following hardware: Processor Intel Core i5-4460 $3.2 \mathrm{GHz}$, with $8 \mathrm{~GB}$ of DDR3 memory; the genetic algorithm was developed in the Java programming language; to obtain the exact results for the benchmarks described in the previous sections, the optimization software FICO Xpress 7.9 (64bit, release 2015) was used and the experiments were run on a mobile workstation equipped with a processor 


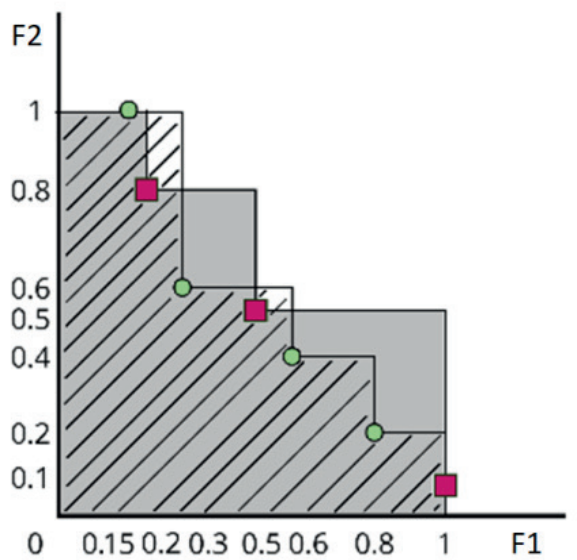

Figure 2 Example of the two compared Pareto fronts,

the first surface area of the Pareto front (area: 0.6) is marked in hatched,

the second (area: 0.69) in grey; it is assumed, that the lower value is better so the first front is the better one [20]

Table 1 Comparation of areas under normalized exact and approximate Pareto-fronts

\begin{tabular}{cccccc}
\hline & \multirow{2}{*}{ exact result } & \multicolumn{2}{c}{ heuristic GA - one by one } & \multicolumn{2}{c}{ heuristic GA - each by each } \\
\cline { 3 - 5 } & & average & best result & average & best result \\
\hline BA & 0.15854043 & 0.346925 & 0.20727 & 0.336714 & 0.233965 \\
BB & 0.060582 & 0.557023 & 0.382774 & 0.549060 & 0.360898 \\
KE & 0.069284634 & 0.367419 & 0.218435 & 0.365578 & 0.200383 \\
NR & 0.1614823 & 0.454372 & 0.329542 & 0.437669 & 0.304113 \\
PO & 0.045180031 & 0.543496 & 0.396628 & 0.4419 & 0.34113 \\
TN & 0.058402242 & 0.401947 & 0.298893 & 0.379348 & 0.224277 \\
TT & 0.043653727 & 0.441135 & 0.132829 & 0.518205 & 0.294873 \\
ZA & 0.054469734 & 0.605164 & 0.446484 & 0.592686 & 0.419366 \\
\hline
\end{tabular}

Intel Core i5-7200U $2.5 \mathrm{GHz}$ and $16 \mathrm{~GB}$ of DDR4 RAM.

Eight regions of the Slovak Republic were used as the benchmarks. For each self-governing region, i.e. Bratislava (BA), Banska Bystrica (BB), Kosice (KE), Nitra (NR), Presov (PO), Trencin (TN), Trnava (TT) and Zilina (ZA), all cities and villages were taken into account, as the set of possible locations. The cardinalities of these sets vary from 87 to 664 according to the considered region.

It was necessary to use an effective way how to compare two solutions (or more), which are represented by the semi-Pareto fronts. Both compared fronts needed to be normalized, so that all the solutions in the front have both objective functions ranging from 0 to 1 . Comparison of more than two fronts can be also done using this approach. After the mentioned transformation one can calculate the area restricted by the axes and curve of Pareto front. The points of the individual Pareto front and semi Pareto front are connected by the line as in Figure 2. Both areas under the connector were calculated. The better approximation of the Pareto front corresponds to one with the smaller area [20].

For each of the eight regions, the parameter settings, according to two approaches, were obtained. The timeconsuming heuristic run was performed, where 40 different semi-Pareto fronts were obtained for each region and each parameter combination.

The exact results were compared to the two heuristic approaches with initial parameters obtained in different ways. "One by one" and "Each by each" are the parameter tuning approaches.

The first parameter tuning approach, called "One by one" parameter, consists of the following steps. Firstly, the set of $r$ parameters is ordered into a sequence $p_{1}, p_{2}$, $\ldots, p_{r}$ and subscript $t$ of the current parameter is set to 1. The parameters are set at some initial values from their ranges. Then, in the $t$-th step, one has to find the best value of parameter $p$. The set of permissible values is given for each parameter. The best option is selected according to the results of the test run. The best value of the parameter is assigned to $p_{t}$ for the next step. Finally, if $t$ is less than $r$, the $t$ is increased by one and the process continues. Otherwise, the tuning process terminates.

The second tuning process is called "Each by each" and consists of a performed time-consuming enumeration of all the combinations of individual parameters.

The following parameters were tuned: population size, elite set size ratio, mutation probability and tournament size ratio. The other used parameters with 


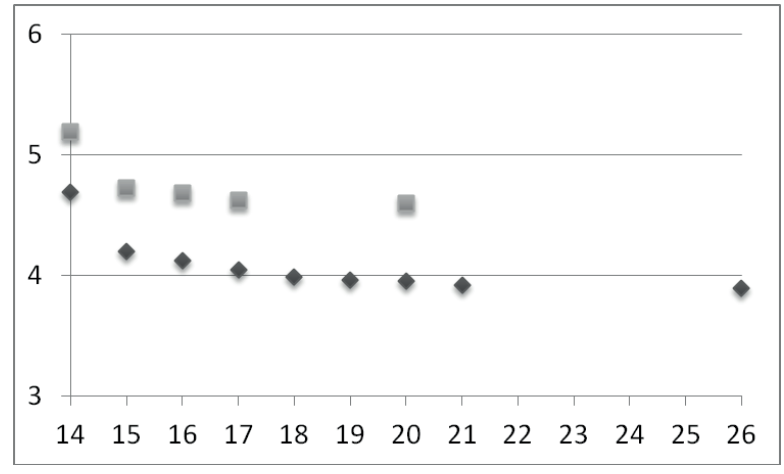

Figure 3 Exact Pareto front (rhombus) compared to heuristic one (squares) for the Banska Bystrica region

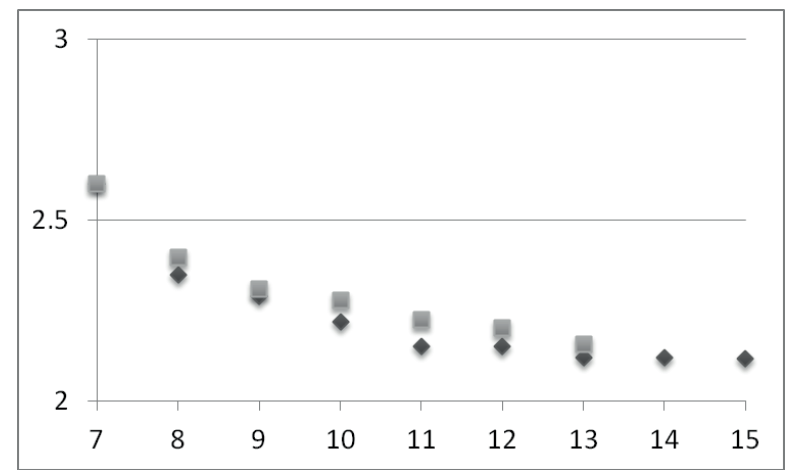

Figure 4 Exact Pareto front (rhombus) compared to heuristic one (squares) for the Bratislava region

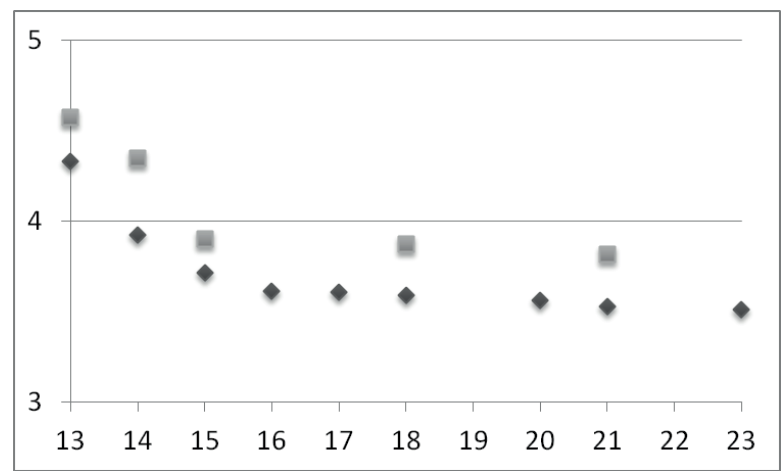

Figure 5 Exact Pareto front (rhombus) compared to heuristic one (squares) for the Kosice region

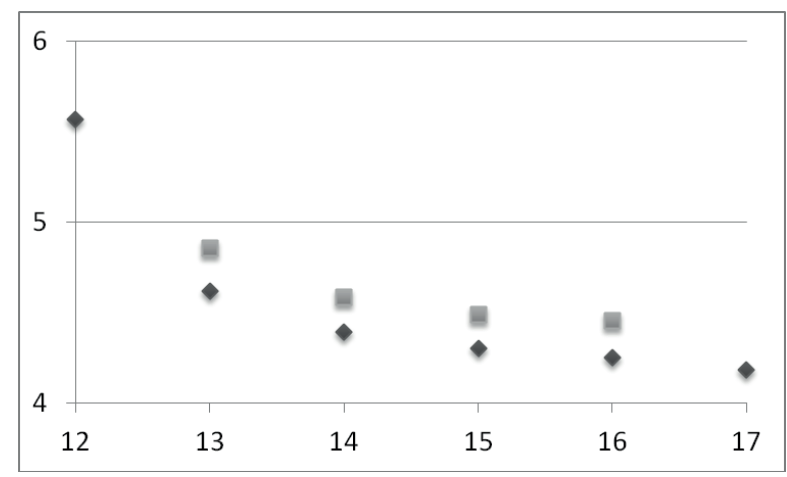

Figure 6 Exact Pareto front (rhombus) compared to heuristic one (squares) for the Nitra region

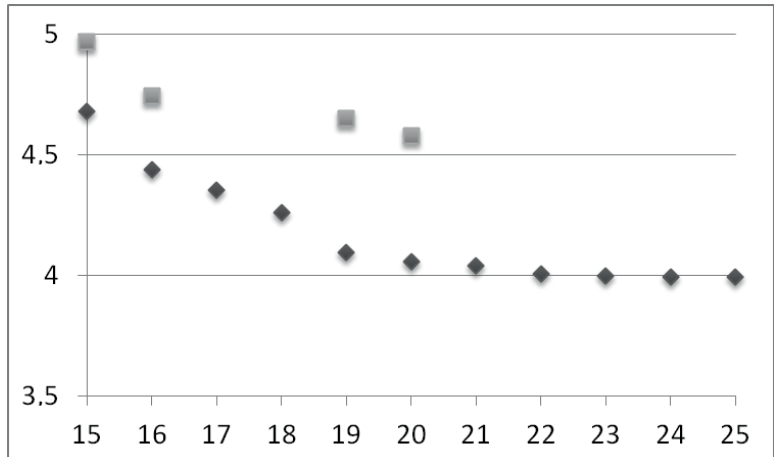

Figure 7 Exact Pareto front (rhombus) compared to heuristic one (squares) for the Presov region

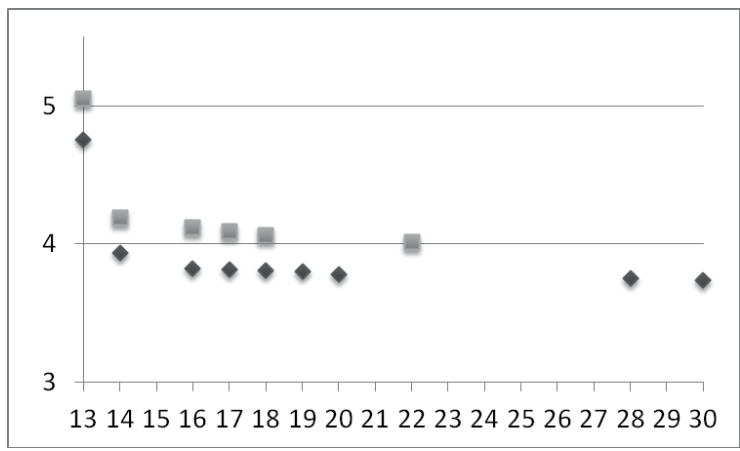

Figure 8 Exact Pareto front (rhombus) compared to heuristic one (squares) for the Trencin region

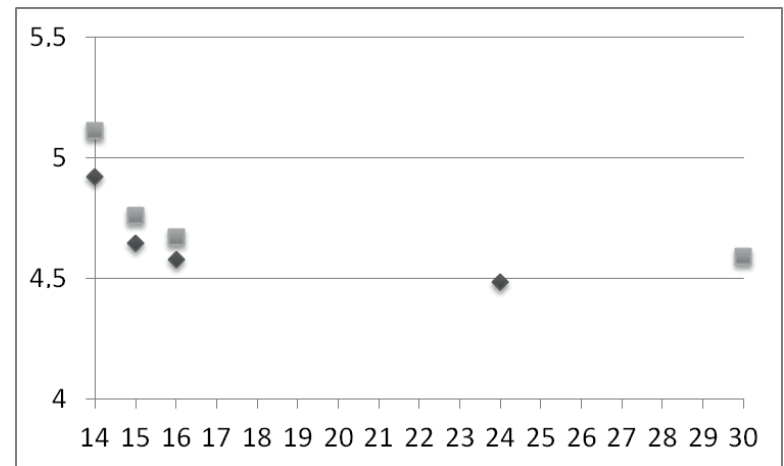

Figure 9 Exact Pareto front (rhombus) compared to heuristic one (squares) for the Trnava region

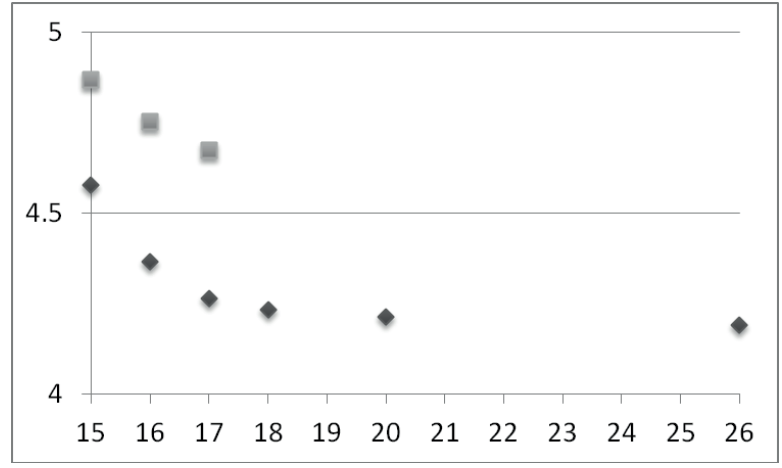

Figure 10 Exact Pareto front (rhombus) compared to heuristic one (squares) for the Zilina region 
fixed values: Mutation step, maximal mutation count per offspring, offspring optimization probability, the acceptable difference in the population and final count of the population diversity controls.

The hypothesis that mean values for both sets of heuristic results are the same as the optimal result was tested. The hypotheses were tested using the Student t-test. To use this test, it was necessary to verify that the results obtained had a normal distribution. The Kolmogorov-Smirnov test for normality was used for that purpose. As it is shown in Table 1, the heuristic results were compared to the exact ones. After performing the above-mentioned test, the hypotheses that mean values of a heuristic run are the same as an exact result at $5 \%$ significance level for all the datasets, were rejected.

Comparison of the best-found approximation of the Pareto fronts and exact Pareto fronts, for each selfgoverning region, can be found in Figures $3-10$. The horizontal axis depicts the min-max values in $\mathrm{km}$ and the vertical axis contains average min-sum values in $\mathrm{km}$ of resulting Pareto fronts.

According to the presented results in Figures 3-10 and Table 1, the genetic algorithm can be used for approximation of the Pareto front. One can see that in Bratislava, Kosice, Nitra, Trencin and Trnava (Figures 4, 5, 8 and 9) relatively good approximation of the exact results was obtained, which means that this approach can be permissible while solving large problems. However, in general comparison to the exact results, one can see that more numerous Pareto front members were found using the exact approach.

\section{Conclusions}

This research was devoted to inspection of the genetic algorithm ability in obtaining an approximation of the Pareto front of the p-location problem solutions. For this purpose, two conflicting criteria were considered. The first min-sum objective was to minimize an average distance between a user and the nearest service center and the other min-max objective was to minimize the maximal distance from a user to the nearest service center. To be able to perform proper analysis of the approximate results, the exact Pareto front were determined for each benchmark, by an exact optimization method and comparing it to the Pareto front approximation, authors came to the following findings. The genetic algorithm can be used to find an approximation of the Pareto front of the non-dominated public service system designs with conflicting criteria, but the approximation may be a bit biased in the case when a bigger instance of the problem is solved. A future research in this field will be aimed at methods, which can enable to direct the evolutionary process at a refinement of the Pareto front approximation.

\section{Acknowledgement}

This work was supported by the Slovak Research and Development Agency under the Contract no. APVV19-0441 and by the by the research grant VEGA 1/0216/21 "Design of emergency systems with conflicting criteria using artificial intelligence tools".

\section{References}

[1] BROTCORNE, L., LAPORTE, G., SEMET, F. Ambulance location and relocation models. European Journal of Operational Research [online]. 2003, 147(3), p. 451-463. ISSN 0377-2217. Available from: https://doi.org/10.1016/ S0377-2217(02)00364-8

[2] DOERNER, K. F., GUTJAHR, W. J., HARTL, R. F., KARALL, M., REIMANN, M. Heuristic Solution of an Extended Double-Coverage Ambulance Location Problem for Austria. Central European Journal of Operations Research. 2005, 13(4), p. 325-340. ISSN 1435-246X, eISSN 1613-9178.

[3] JANOSIKOVA, L. Emergency medical service planning. Communications - Scientific Letters of the University of Zilina [online]. 2007, 9(2), p. 64-68. ISSN 1335-4205, eISSN 2585-7878. Available from: http://komunikacie. uniza.sk/index.php/communications/article/view/1133

[4] GARCIA, S., LABBE, M., MARIN, A. Solving large p-median problems with a radius formulation. INFORMS Journal on Computing [online]. 2011, 23(4), p. 546-556. ISSN 1091-9856, eISSN 1526-5528. Available from: https://doi.org/10.1287/ijoc.1100.0418

[5] KVET, M. Computational study of radial approach to public service system design with generalized utility. In: 10th International IEEE Conference Digital Technologies 2014: proceedings. 2014. p. 198-208.

[6] KVET, M. Advanced radial approach to resource location problems. In: Studies in computational intelligence: developments and advances in intelligent systems and applications [online]. ROCHA, A., REIS, L. P. (eds.). Springer, 2018. ISBN 978-3-319-58965-7, eISBN 978-3-319-58965-7, p. 29-48. Available from: https://doi.org/10.1007/978-3-319-58965-7

[7] OGRYCZAK, W., SLIWINSKI, T. On direct methods for lexicographic min-max optimization. In: International Conference on Computational Science and Its Applications ICCSA 2006: proceedings [online]. Lecture Notes in Computer Science. Vol. 3982. Berlin, Heidelberg: Springer, 2006. ISBN 978-3-540-34075-1, eISBN 978-3-540-34076-8, p. 802-811. Available from: https://doi.org/10.1007/11751595_85 
[8] JANACEK, J., KVET, M. Public service system design with disutility relevance estimation. In: International Conference on Mathematical Methods in Economics 2013: proceedings. 2013. p. 332-337.

[9] ELLOUMI, S., LABBE, M., POCHET, Y. A new formulation and resolution method for the p-center problem. INFORMS Journal on Computing [online]. 2004, 16, p. 84-94. ISSN 1091-9856, eISSN 1526-5528. Available from: https://doi.org/10.1287/ijoc.1030.0028

[10] SAYAH, D., IRNICH, S. A new compact formulation for the discrete p-dispersion problem. European Journal of Operational Research [online]. 2016, 256(1), p. 62-67. ISSN 0377-2217. Available from: https://doi.org/10.1016/j. ejor.2016.06.036

[11] KVET, M., JANACEK, J. Price of fairness in public service system design. In: International Conference on Mathematical Methods in Economics 2014: proceedings. 2014. p. 554-559.

[12] KVET, M., JANACEK, J. Lexicographic fair design of robust emergency service system. In: International Conference on Mathematical Methods in Economics 2016: proceedings. 2016. p. 495-500

[13] BERTSIMAS, D., FARIAS, V. F., TRICHAKIS, N. The price of fairness. Operations Research [online]. 2011, 59(1), p. 17-31. ISSN 0030-364X, eISSN 1526-5463. Available from: https://doi.org/10.1287/opre.1100.0865

[14] JANOSIKOVA, L., GABRISOVA, L., JEZEK, B., Load balancing location of emergency medical service stations. E+M Ekonomie a Management / E+M Economics and Management [online]. 2015, 18(3), p. 30-40. ISSN 1212-3609, eISSN 2336-5064. Available from: http://dx.doi.org/10.15240/tul/001/2015-3-003

[15] ARROYO, J. E. C., DOS SANTOS, P. M., SOARES, M. S., SANTOS, A, G. A multi-objective genetic algorithm with path relinking for the p-median problem. In: Ibero-American Conference on Artificial Intelligence IBERAMIA 2010: proceedings [online]. Lecture Notes in Computer Science. Vol. 6433. Berlin, Heidelberg: Springer, 2010. ISBN 978-3-642-16951-9, eISBN 978-3-642-16952-6, p. 70 - 79. Available from: https://doi.org/10.1007/978-3-64216952-6_8

[16] JANACEK, J. Public service system design with fuzzy parameters of perceived utility. In: Central European Journal of Operations Research [online]. 2015, 23(3), p. 595-606. ISSN 1435-246X, eISSN 1613-9178. Available from: https://doi.org/10.1007/s10100-014-0370-7

[17] KVET, M., JANACEK, J. Iterative approaches to design of robust emergency system. In: 2017 IEEE International Conference on Service Operations and Logistics and Informatics SOLI: proceedings. 2017. ISBN 978-1-53860949-1, p. 13-16.

[18] RYBICKOVA, A., BURKETOVA, A., MOCKOVA, D. Solution to the location-routing problem using a genetic algorithm. In: 2016 Smart Cities Symposium Prague SCSP: proceedings [online]. IEEE. 2016. ISBN 978-1-50901116-2, p. 1-6. Available from: https://doi.org/10.1109/SCSP.2016.7501016

[19] RYBICKOVA, A., MOCKOVA, D., TEICHMANN, D. Genetic algorithm for the continuous location-routing problem. Neural Network World [online]. 2019, 29, p. 173-187. ISSN 1210-0552, eISSN 2336-4335. Available from: https://doi.org/10.14311/NNW.2019.29.012

[20] GRYGAR, D., FABRICIUS, R. An efficient adjustment of genetic algorithm for pareto front determination. Transportation Research Procedia [online]. 2019, 40, p. 1335-1342. ISSN 2352-1465. Available from: https://doi.org/10.1016/j.trpro.2019.07.185

[21] REEVES, C. R. Genetic algorithms. In: Handbook of Metaheuristics. 2. ed. GENDREAU, M., POTVIN, J. Y. (eds.). New York, Dordrecht, Heidelberg, London: Springer, 2010. ISBN 978-3-319-91086-4. 